Check for updates

Cite this: RSC Adv., 2019, 9, 37614

Received 9th July 2019

Accepted 13th November 2019

DOI: $10.1039 / c 9 r a 05243 g$

rsc.li/rsc-advances

\section{Probing hemoglobin glyco-products by fluorescence spectroscopy}

\begin{abstract}
Aristos loannou (D) and Constantinos Varotsis*
Maillard reaction products (MRPs) participate in reactions of carbohydrate intermediates with proteins, resulting in the formation of advanced glycation end-products (AGEs). Dietary Maillard reaction products are recognized as potential chemical modifiers of human proteins. We have investigated the reaction of isolated MRPs from an asparagine-glucose model system with hemoglobin $(\mathrm{Hb})$ to elucidate the binding effect of the MRPs in hemoglobin by fluorescence spectrophotometry. The tryptophan-specific fluorescence obtained for glycated hemoglobin exhibited a Stokes effect since the wavelength of the emission peak was shifted to a higher wavelength than that of native $\mathrm{Hb}$. The formation of new fluorescence emission features indicates the formation of modified hemoglobin species. Fluorescence spectroscopic studies provide evidence that the conformational changes in the $\beta$-Trp 37 moiety induce motion of the distal His 64 (E7) in the heme binding pocket. This results in the formation of inactive hemichrome forms of hemoglobin which are related to blood disorders.
\end{abstract}

\section{Introduction}

Maillard Reaction Products (MRPs) are attributes of thermally processed foods, contributing to their flavor, aroma and texture..$^{1-4}$ The increased consumption of MRPs in recent years has pointed towards their implication in the development of processes such as diabetes and degenerative diseases. Advanced glycation end products (AGEs) lead to the formation of unstable, reactive intermediates that readily form intra- and intermolecular covalent crosslinks or glyco-oxidation products involved in the vascular and renal complications of diabetes mellitus. ${ }^{5}$ People with diabetes and renal disease display elevated serum AGE levels and reduced urinary AGE excretion. Unreactive AGEs can readily form new crosslinks with low density lipoprotein (LDL) or collagen. It has been demonstrated that AGEs absorbed into the blood stream may represent a major source of chemically and biologically active toxins. These glycotoxins are only partially eliminated in the urine and may exert significant reactivity in the body. Persons with impaired renal functions are unable to efficiency excrete glycotoxins in the urine, resulting in abnormally high AGE concentrations in the blood and tissues. Hemoglobin is the most abundant blood protein and consists of two $\alpha$ and $\beta$ subunits which are non-covalently associated within erythrocytes as a $64.5 \mathrm{kDa}$ tetramer. ${ }^{5}$ The natural mechanisms of $\mathrm{Hb}$ uptake and decomposition, the latter of which leads to unloading of any bound compounds, can be employed to selectively monitor the Hb-bound complexes.

Cyprus University of Technology, Department of Environmental Science and Technology, Limassol, Cyprus. E-mail: c.varotsis@cut.ac.cy; Fax: +357 25002802
Recently, the isolation of reaction products of asparagine with reducing sugars at high $\mathrm{pH}$ and temperature was explored by high performance liquid chromatography (HPLC) coupling methods. ${ }^{6-8}$ Spectroscopic analysis of selected Maillard reaction products demonstrated the appearance of asparaginesaccharide conjugates. ${ }^{7}$ These were shown to induce the formation of a hemichrome species in hemoglobin, which obstructs the ligand binding site irreversibly. ${ }^{8}$ The intrinsic fluorescence of tryptophans (W) and tyrosines (Y) in $\mathrm{Hb}$ has been used as an important probe in understanding the dynamics and conformational changes induced by ligand binding and to elucidate the binding location and relevant parameters related to binding interactions. ${ }^{9-11}$ Excitation with light at $280 \mathrm{~nm}$ reflects contribution from both $\mathrm{W}$ and $\mathrm{Y}$ residues whereas excitation at $296 \mathrm{~nm}$ is used to distinguish the contribution of $\mathrm{W}$ residues alone without any fluorescence energy transfer from the $\mathrm{Y}$ residues. The assignment of $\beta 37$ Trp as the origin of the emitted fluorescence in $\mathrm{Hb}$ when excited by light at $296 \mathrm{~nm}$ has been established providing solid evidence that this spectral feature is sensitive to conformational changes in Hb. ${ }^{9-11}$ Anomalous emissions upon excitation at $280 \mathrm{~nm}$ have been attributed to a combination of tyrosine and tyrosinate emissions. Tryptophan fluorescence measurements provide a reliable analytical tool to characterize the tryptophan environment which is related to the tertiary conformation. A rigid environment around the Trp and a change in polarity affects the emission of the Trp fluorescence spectrum. A blue shift in the fluorescence emission indicates the presence of a hydrophobic environment around the Trp and increased fluorescence intensity indicates a more rigid environment. ${ }^{\mathbf{9 1 0}}$ The fluorescence of advanced Maillard 
reaction products has also been investigated and the potential application in food sciences has been reported. ${ }^{12-15}$

In this study, we decided to use fractions of an asparagine/ carbohydrate system to glycate hemoglobin in order to demonstrate that other glycated species may contribute towards the pool of glycated proteins in the blood. The reaction of the discrete MRPs originated from an asparagine-glucose model system with hemoglobin was investigated after one day and one month incubation time by fluorescence excitation/emission spectrophotometry. These specific time points were selected to demonstrate short and long-term exposure, respectively, of hemoglobin to these glycated species. The Trp-specific fluorescence obtained for the glycated-Hb complexes exhibited Stokes effect which is attributed to the formation of $\mathrm{Hb}$-MRPs complexes and to structural modification of $\mathrm{Hb}$ induced by the MRPs. We propose that the MRPs interact through hydrophobic and hydrogen bonding in the moiety of $\beta-37$ Trp, and the interaction perturbs the structural and functional properties of $\mathrm{Hb}$. We suggest that the conformational changes at the $\mathrm{N} 1 \mathrm{H}$ site have a direct effect in the $\mathrm{O}_{2}$ binding site forming a hemichrome, and thus, $\beta-37$ Trp may be useful as an intrinsic probe to study the dynamic processes involved in the formation of hemichromes which are related to blood disorders. ${ }^{5,8}$ Furthermore, comparison of the fluorescence data in the presence and absence of the MRPs with UV resonance Raman data indicate that the microenvironment of $\beta 37$ Trp which includes $\alpha$ Tyr140 which is located in the microenvironment of $\beta 37 \mathrm{Trp}, \alpha 43 \mathrm{Tyr}$, and $\beta 145$ Tyr is involved in the $\mathrm{R} \rightarrow \mathrm{T}$ transition. ${ }^{8}$

\section{Experimental}

\subsection{Sample preparation of MRPs}

Solutions of glucose and asparagine $(0.2 \mathrm{M}, \mathrm{pH} 8.0)$ were prepared in phosphate buffer ( $50 \mathrm{mM}, \mathrm{pH} 8.0)$. Samples were heated in closed screw-capped tubes at $180^{\circ} \mathrm{C}$ in a heating oven (Memmert, Germany) for 2 hours.

\subsection{HPLC-fraction collector analysis of MRPs}

The HPLC instrumental setup and the separation of individual MRP fractions were described in detail earlier., ${ }^{7,8}$ The HPLC experimental setup consisted of a Varian 218 Prepstar Solvent Delivery Module, an Agilent Manual FL-Injection Valve, an Agilent 1260 Infinity Variable Wavelength Detector (VWD) and an Agilent 440 LC Fraction Collector. HPLC analysis was performed on a $4.6 \times 250 \mathrm{~mm}, 5 \mu \mathrm{m}$ particle size, Zorbax SB-Aq analytical column (Agilent Technologies). Maillard reaction products (MPRs) were analyzed under aqueous conditions using a previously reported method. ${ }^{7}$ Water at a flow rate of $0.5 \mathrm{ml} \mathrm{min}^{-1}$ was used isocratically as the mobile phase at room temperature. Maillard reaction products (MPRs) were detected at $200 \mathrm{~nm}$.

\subsection{HPLC-fraction collector analysis of MRPs}

Hemoglobin from bovine blood (Sigma) at approximately $1 \mathrm{mM}$ concentration in $50 \mathrm{mM}$ phosphate buffer ( $\mathrm{pH} 8.0$ ) was diluted hundred-fold to avoid inner filter effects during fluorescence measurement. The diluted hemoglobin was immediately mixed with each individual LC fraction $(2: 1 \mathrm{v} / \mathrm{v})$ from the reaction between asparagine and glucose at $180{ }^{\circ} \mathrm{C}$. The solutions were mixed and kept at room temperature. The fluorescence spectra of the mixtures were collected up to 30 days incubation.

\subsection{Fluorescence analysis}

The Cary Eclipse Fluorescence Spectrophotometer (Agilent Technologies, USA) controlled from a PC using the Agilent Technologies WinFLR software (version 1.2) was used for obtaining the fluorescent signal. Fluorescence values of the native and glycated hemoglobin samples were measured using a clear four-sided quartz cuvette in the spectrophotometer sample compartment. The excitation and emission slit widths for both the excitation and emission monochromators were set with the value of $5.0 \mathrm{~nm}$. The Excitation-Emission Matrix (EEM) measurement and processing were generated by scanning emission spectra from $320 \mathrm{~nm}$ to $500 \mathrm{~nm}$ at $1 \mathrm{~nm}$ intervals, with $5 \mathrm{~nm}$ increments of the excitation wavelengths from $260 \mathrm{~nm}$ to $310 \mathrm{~nm}$. The Milli-Q water blank was subtracted from the sample EEM spectra.

\section{Results and discussion}

Maillard reactions are highly influenced by $\mathrm{pH}$ and temperature. ${ }^{4}$ Model systems similar to reactions naturally occurring in processed foods that have undergone high temperature treatments are useful in resembling more complex chemical systems occurring in foods. Maillard reaction rate is highly increased by the combination of factors such as slightly alkaline $\mathrm{pH}$ and high temperatures. $^{7,8}$ In this context, we have chosen $\mathrm{pH} 8$ and a temperature of $180{ }^{\circ} \mathrm{C}$ in order to produce these Maillard reaction intermediates in sufficient concentrations for subsequent isolation. Our choice of $\mathrm{pH}$ aimed to keep a balance between our model Maillard system and the physiological pH of hemoglobin in human blood which is slightly alkaline.

The HPLC chromatograms of the asparagine-glucose reactions are shown in Fig. 1. A major signal at Rt $=5.7 \mathrm{~min}$ is present which corresponds to the excess unreacted asparagine and other smaller chromatographic peaks arising from individual MRPs. Fraction 1 corresponds to the Schiff base and Fractions 2, 3 and 4 have been characterized to originate from the Amadori product, decarboxylated Amadori product and
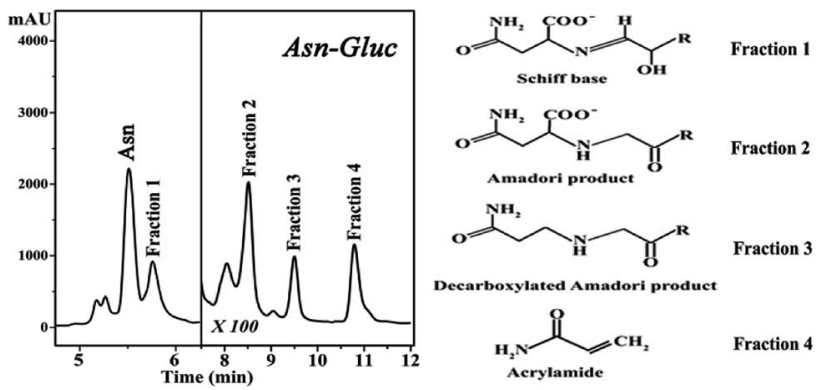

Fig. 1 High performance liquid chromatography (HPLC) chromatograms of the reaction mixture of asparagine and glucose $(\mathrm{pH} 8.0,180$ $\left.{ }^{\circ} \mathrm{C}\right)$. 
acrylamide, respectively. ${ }^{7}$ These fractions were incubated with $\mathrm{Hb}$ in a one day and one month period and their effect on hemoglobin was probed by fluorescence spectroscopy.

Proteins react with glucose to form a stable Amadori product through Schiff base adducts that are transformed to advanced glycation end products (AGEs) some of which exhibit fluorescence. ${ }^{\mathbf{1 4 - 1 6}}$ Therefore, their fluorescence intensities indicate of the extent of AGE-modified proteins. These excitation-emission maxima are distinct from tryptophan fluorescence in proteins. A worthy feature of intrinsic protein fluorescence in $\mathrm{Hb}$ is the high sensitivity of the local environment of $\beta-37$ Trp. ${ }^{9-11}$ Alterations in the emission spectra of tryptophan may occur in response to conformational transitions, subunit association, substrate binding, and/or denaturation.

Fig. 2 shows the fluorescence excitation-emission matrix (EEM) of native hemoglobin ( $\mathrm{pH}$ 8.0). Hb displays weak fluorescence under these conditions when maximally excited at $280 \mathrm{~nm}$. The contour map enables the identification of peaks within a multi-component sample matrix. The different colors are associated with intensity with the deep red being the most intense. It is evident that for native hemoglobin the maximum excitation/emission is $280 \mathrm{~nm} / 338 \mathrm{~nm}$ (Fig. 2). Excitation at $280 \mathrm{~nm}$ will excite both tyrosine and tryptophan residues. The signals from the $280 \mathrm{~nm}$ excitation are three times more intense and thus small changes can be determined more reliably. The fluorescence spectra shown in Fig. 2 are in good agreement with those previously reported., ${ }^{9,10}$ Upon excitation at $296 \mathrm{~nm}$, the Trp residues are selectively excited and the majority of the fluorescence observed in $\mathrm{Hb}$ has been previously assigned to the $\beta-37$ Trp residue. The observed difference in fluorescence intensity between the $\mathrm{T}$ and $\mathrm{R}$ states in $\mathrm{Hb}$ has been attributed to the changes occurring at $\beta-37$ Trp. Therefore, the Trp-37 in the $\beta$ chains of $\mathrm{Hb}$ is the fluorophoric moiety of $\mathrm{Hb}$ and can serve as the marker residue for monitoring the dynamics of $\mathrm{Hb}$ complexes. It is known that $\alpha-140$ Tyr which is located in the microenvironment of $\beta-37$ Trp, $\beta-42$ Tyr, and $\beta-145$ Tyr undergo conformational changes during the $\mathrm{R} \rightarrow \mathrm{T}$ transition. A dramatic change in fluorescence intensity could result from altered Tyr $\rightarrow$ Trp and Trp $\rightarrow$ heme energy-transfer pathways., ${ }^{\mathbf{9} 10}$

Fig. 3 shows the fluorescence emission spectra of the reactions of $\mathrm{Hb}$ with the Schiff base (Fraction 1), Amadori product (Fraction 2), decarboxylated Amadori product (Fraction 3) and

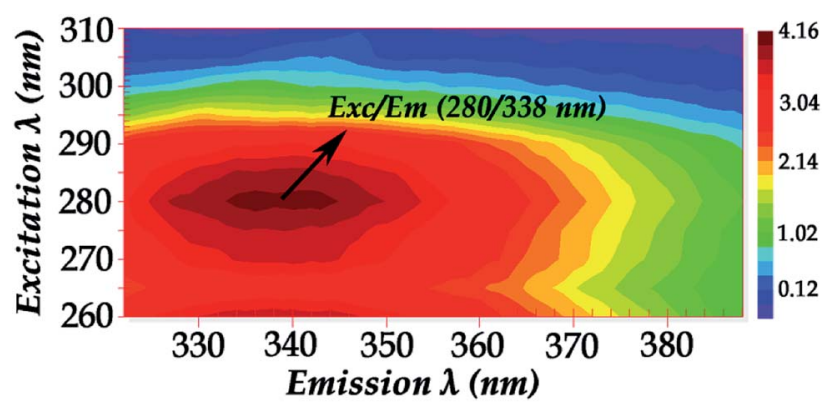

Fig. 2 Fluorescence excitation-emission matrix (EEM) of native hemoglobin ( $\mathrm{pH} 8.0$ ) (excitation wavelength range: $260-310 \mathrm{~nm}$ ).
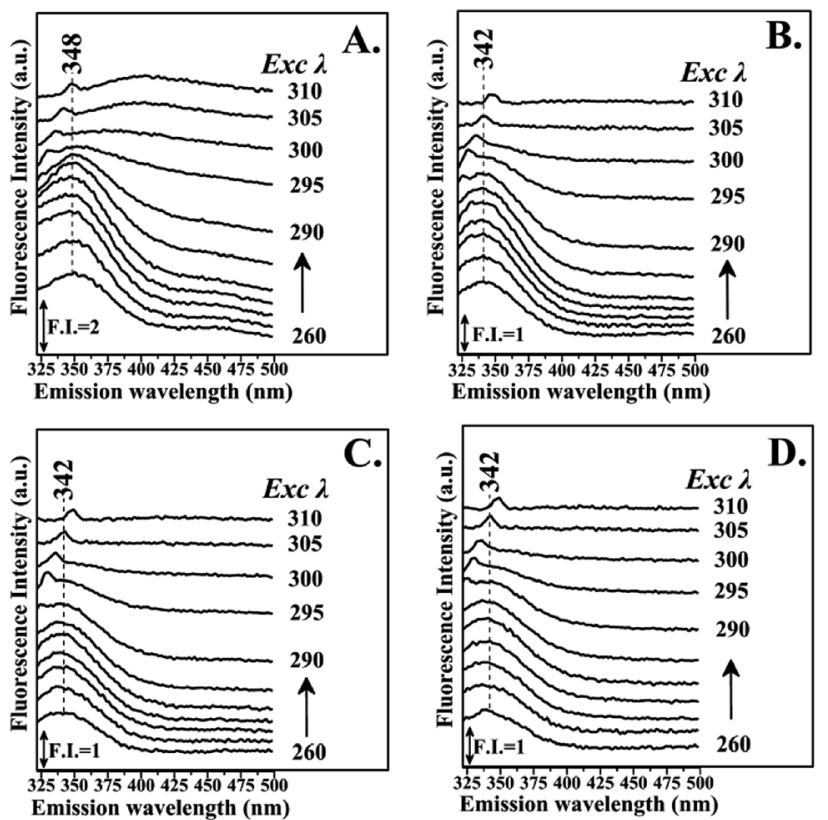

Fig. 3 Fluorescence emission spectra of hemoglobin with: Panel A: LC Fraction 1, Panel B: LC Fraction 2, Panel C: LC Fraction 3, Panel D: LC Fraction 4 from the reaction of Asn-Gluc after 1 day incubation (excitation wavelength range: $260-310 \mathrm{~nm}$ ).

acrylamide (Fraction 4) after 1 day of incubation. The Raman peak of $\mathrm{H}_{2} \mathrm{O}$ which is present in all the fluorescence spectra is always located of $3400-3600 \mathrm{~cm}^{-1}$ lower in energy than the excitation wavelength. In the $260-295 \mathrm{~nm}$ excitation range, the emission maxima were located at $348 \mathrm{~nm}$ (Fig. 3A) for the Schiff base (Fraction 1) and approximately at $342 \mathrm{~nm}$ (Fig. 3B-D) for the Amadori product (Fraction 2), decarboxylated Amadori product (Fraction 3) and acrylamide (Fraction 4). The fluorescence obtained for these glycated $\mathrm{Hb}$ complexes exhibited a Stokes effect since the wavelength of the emission peak is shifted $10 \mathrm{~nm}$ in the Hb-Schiff base complex and $4 \mathrm{~nm}$ in the Hb-Amadori complex, Hb-decarboxylated Amadori product and $\mathrm{Hb}$-acrylamide complexes. The lowering of this energy level can be associated to conformational changes of $\mathrm{Hb}$. From our experimental results after 1 day incubation it was evident that all 4 fractions achieve the initial binding to hemoglobin as there is a conformational change in the hemoglobin molecule as depicted by the fluorescence spectral shifts. The origin of the fluorescence when excited by light at $280 \mathrm{~nm}$ is attributed to tyrosine and tryptophan emissions. The decreased intensity that accompanied the emission shift serves as evidence that this conformational change arises from $\beta-37$ Trp at the $\alpha_{1} \beta_{2}$ interface when the Hb-complexes are excited by light at $296 \mathrm{~nm} .{ }^{\mathbf{1 0}}$ No emissions are observed in the $300-310 \mathrm{~nm}$ excitation range. It is generally accepted that when the tryptophan residue becomes hydrogen bonded or increasingly exposed to water, its fluorescent emission shifts to longer wavelengths. Therefore, the increased hydrophilicity can arise as a result of a conformational change coupled to increased exposure of $\beta-37$ Trp to the solvent. The observed behavior of the $\mathrm{Hb}$ complexes with respect to the contribution of the $\beta-37$ Trp environment at the 
$\alpha_{1} \beta_{2}$ interface is similar to that observed during the $\mathrm{R} \rightarrow \mathrm{T}$ transition.,10 Fig. 4 illustrates the fluorescent spectra of the hemoglobin-MRP mixtures after 1 month incubation. There is a noticeable shift to longer wavelengths in the emission spectra of the Hb-Amadori complex, Hb-decarboxylated Amadori product and $\mathrm{Hb}$-acrylamide complexes as compared to those spectra observed after one day of incubation, shown in Fig. 3B$\mathrm{D}$, whereas that of the $\mathrm{Hb}$-Schiff base complex does not show any further change in fluorescent characteristics as that observed in the spectra in Fig. 3A after one day of incubation. This may denote that there was no significant $\mathrm{Hb}$-AGE formation in this case. On the contrary, for the other 3 fractions there are further fluorescence shifts after this time frame, demonstrating further structural change in hemoglobin and Hb-AGE formation. The $6 \mathrm{~nm}$ shift of the $\mathrm{Hb}$-Amadori complex and $\mathrm{Hb}-$ decarboxylated Amadori product indicates that prolonged incubation times induce a further conformational change at the $\beta-37$ Trp microenvironment and the $11 \mathrm{~nm}$ shift of the $\mathrm{Hb}-$ acrylamide complex indicates strong $\mathrm{H}$-bonded interactions and conformational changes in the moiety of the $\beta-37$ Trp residue. The appearance of a new strong emission feature around $395 \mathrm{~nm}$ at the excitation range of $295-310 \mathrm{~nm}$ is attributed to fluorescence quenching effect on tryptophan fluorescence. This effect was often utilized to demonstrate ligand-protein interactions. ${ }^{16}$ A similar $390 \mathrm{~nm}$ emission band was recently identified from fluorescent intensity maps of degenerated cartilage from osteoarthritis patients. The authors discussed that this was a result of non-enzymatic glycation reaction which is followed by the formation of several types of fluorescent crosslinks (AGEs). ${ }^{17} \mathrm{Hb}$-AGE fluorescent crosslinks
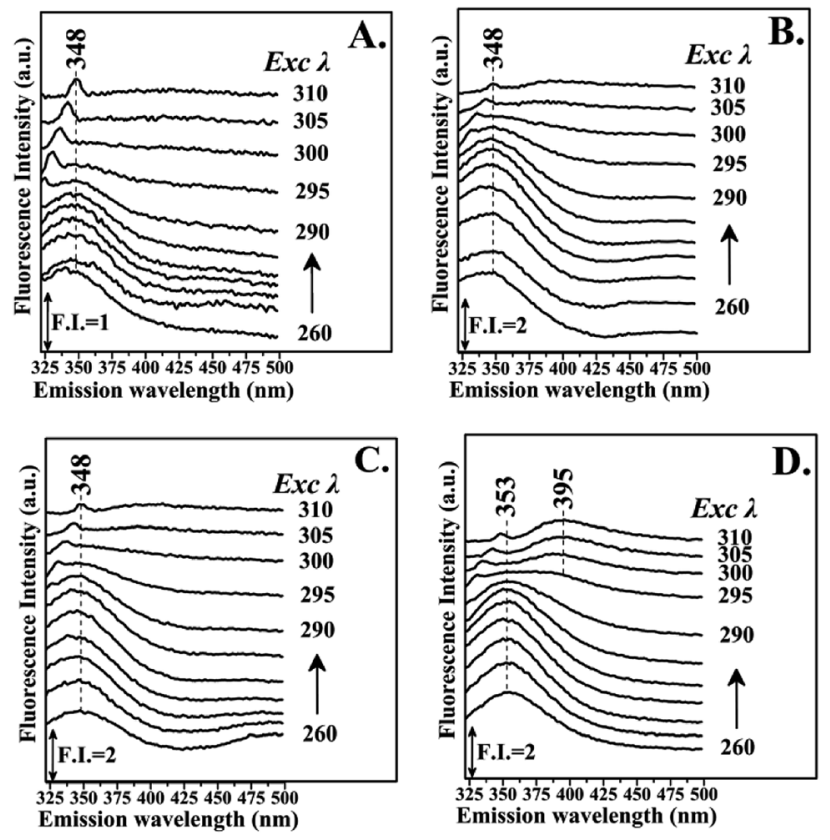

Fig. 4 Fluorescence emission spectra of hemoglobin with: PanelA: LC Fraction 1, Panel B: LC Fraction 2, Panel C: LC Fraction 3, Panel D: LC Fraction 4 from the reaction of Asn-Gluc after 1 month incubation (excitation wavelength range: $260-310 \mathrm{~nm}$ ). are likely to result in loss of protein functionality as it is evident from the $\mathrm{Hb}$-acrylamide fluorescent profile.

Glycation process results in two different products: early and advanced glycation endproducts (AGEs). Hb-AGEs formation is a slower process and occurs after prolonged exposure to glycated species. These events are more predominant after 1 month incubation based on their relative intensities as illustrated by fluorescence mapping of incubated hemoglobin in Fig. 5. In Fig. 5 the fluorescence mapping after one month of
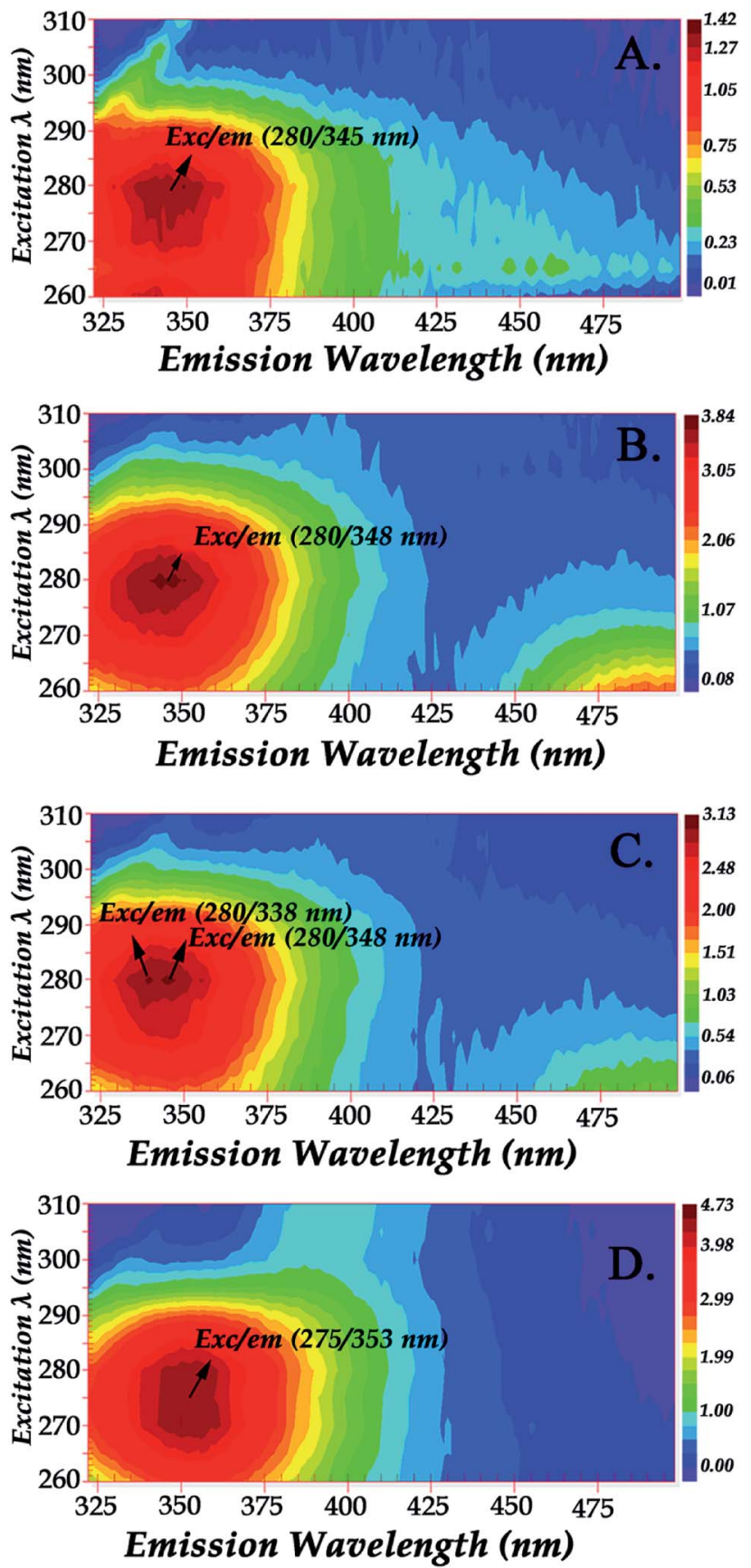

Fig. 5 Fluorescence mapping of hemoglobin with: Panel A: LC Fraction 1, Panel B: LC Fraction 2, Panel C: LC Fraction 3, Panel D: LC Fraction 4 from the reaction of Asn-Gluc after 1 month incubation (excitation wavelength range: $260-310 \mathrm{~nm}$ ). 
incubation of hemoglobin with the LC fractions 1-4 are presented. The fluorescent chemical mapping analysis when compared with the corresponding analysis of $\mathrm{Hb}$ shown in Fig. 2 demonstrates the effect of MRPs in the moiety of $\beta-37$ Trp residue and shows that all the Hb-MRPs complexes are highly heterogeneous systems.

Shifts in fluorescence emission and fluorescence quenching effects are indicative of protein modification in this relatively simple model system. Hb contains three tryptophan residues, but only $\beta-37$ Trp is located at the interface between the two dimers. The $\beta-37$ Trp residues are located in helices $C$ of the $\beta$-subunits and are involved in contacts with the segments FG of the $\alpha$-subunits at the interdimeric $\alpha_{1} \beta_{2}$ and $\alpha_{2} \beta_{1}$ interfaces of the hemoglobin tetramer. ${ }^{18}$ The intrinsic fluorescence signal of $\mathrm{Hb}$ arises from the indole group of tryptophan $\beta-37$ and has been used to determine the changes in the quaternary structure of $\mathrm{Hb}$ upon ligand binding. ${ }^{19}$ In addition, the energetic changes that occur on ligand binding in human hemoglobin are determined by measurements of the exchange rates of the indole proton of $\beta$-37Trp (C3) and the increased exchange rates of the indole proton of $\beta$-37Trp determine ligand binding. The interface regions experience a change in structural free energy upon ligand binding and these hinge regions are involved in the transmission of free energy during its allosteric transition. ${ }^{19}$

Results that involve Trp-37 were also obtained to establish the effectiveness of natural drug delivery mechanisms and investigate the interaction between the drug and it natural carrier Hb. It is thus well established that $\beta-37$ Trp serves as good optical probe for monitoring ligand binding far away $(\sim 15$ A) from the oxygen binding site. ${ }^{11}$

Hemichromes are formed when hemoglobin undergoes conformational changes resulting in the formation of a sixcoordinated $\mathrm{Fe}^{3+}$ low-spin His-Fe-His species. ${ }^{20-22}$ Hemoglobin $\mathrm{A}(\mathrm{HbA})$ in humans can form hemichromes even under physiological conditions as a result of $\mathrm{pH}$ and temperature alterations, and in the autoxidation of oxyHb. ${ }^{20}$ We have recently demonstrated that tetrameric $\mathrm{Hbs}$ form a partial hemichrome state upon addition of MRPs compounds. The results presented here in conjunction with our previous observations strongly indicate that Trp-37 plays a critical role in the formation of the hemichrome..$^{\mathbf{8} 20-22}$ Obviously, the Maillard reaction species act as external ligands to a protein site near the $\beta$-37 Trp moiety, triggering the formation of six-coordinated bishistidyl hemichrome species. ${ }^{23,24}$ The coordination of the distal His 64 (E7) to the iron produces a scissoring motion in helices E and $\mathrm{F}$ which results in modifications in the tertiary structure of the tetramer, mainly in the $\alpha \beta$ interface which is critical in the transition from the $\mathrm{R}$ to the $\mathrm{T}$ state. ${ }^{23}$ Modification of the $\mathrm{EF}$ fragment exposes the heme to a more solvent-exposed position narrowing the heme pocket as the E- and F-helices move toward each other., ${ }^{8,23,24}$

\section{Conclusions}

Maillard reaction products (MRPs) which are absorbed in the blood stream represent a major source of chemically and biologically active toxins. These dietary glycotoxins in high local concentration and time bind irreversibly to $\mathrm{Hb}$. Intrinsic fluorescence activity accompanied by fluorescent mapping was employed in this study to provide experimental evidence regarding the relationship of intrinsic $\mathrm{Hb}$ fluorescence and the chemical events that follow interactions with glycotoxins originated form the Maillard reaction products. The data presented here demonstrate that the primary interactions of the MRPs with $\beta$-37 Trp forming the initial Hb-MRPs complexes are followed by major modifications under prolonged period of time forming the final $\mathrm{Hb}$-MRPs. The present data and those previously reported suggest that $\beta-37$ Trp plays a major role in forming the inactive $\mathrm{Hb}$ hemichrome complexes, as the result of the presence of the Maillard reaction products near the $\beta-37$ Trp moiety of $\mathrm{Hb}$. The implication of the non-enzymatic glycation reaction is the formation of fluorescent cross linked species (AGEs) as a result of the inactive hemichrome form of hemoglobin. ${ }^{25}$ The small number of Maillard reactions being addressed in model reactions in this investigation may point towards the need of a larger in vivo study that will extend the applicability of this fluorescence technique in combination with structure sensitive techniques such as FTIR and Raman spectroscopies to a more heterogeneous in vivo system. ${ }^{26-30}$

\section{Conflicts of interest}

There are no conflicts to declare.

\section{Acknowledgements}

Financial support by the European Regional Development Fund and the Republic of Cyprus through the Research Promotion Foundation (Grant YGEIA/TROFH/0311(BIE)/04) is gratefully acknowledged.

\section{Notes and references}

$1 \mathrm{M}$. Friedman, Food browning and its prevention: an overview, J. Agric. Food Chem., 1996, 44, 631-653.

2 M. Hellwig and T. Henle, Baking, ageing, diabetes: a short history of the Maillard reaction, Angew. Chem., Int. Ed., 2014, 53, 10316-10329.

3 M. D. P. Buera, J. Chirife, S. L. Resnik and G. Wetzler, Nonenzymatic browning in liquid model systems of high water activity: kinetics of color changes due to Maillard's reaction between different single sugars and glycine and comparison with caramelization browning, J. Food Sci., 1987, 52, 1063-1067.

4 E. H. Ajandouz and A. Puigserver, Nonenzymatic browning reaction of essential amino acids: effect of $\mathrm{pH}$ on caramelization and Maillard reaction kinetics, J. Agric. Food Chem., 1999, 47, 1786-1793.

5 T. Koschinsky, C. J. He, T. Mitsuhashi, R. Bucala, C. Liu, C. Buenting, K. Heitmann and H. Vlassara, Orally absorbed reactive glycation products (glycotoxins): An environmental risk factor in diabetic nephropathy, Proc. Natl. Acad. Sci. U. S. A., 1997, 94, 6474-6479. 
6 A. Ioannou and C. Varotsis, Real Time Monitoring the Maillard Reaction Intermediates by HPLC-FTIR, J. Phys. Chem. Biophys., 2016, 6, 210.

7 A. Ioannou, V. Daskalakis and C. Varotsis, Detection of Maillard reaction products by a coupled HPLC-Fraction collector technique and FTIR characterization of $\mathrm{Cu}$ (II)complexation with the isolated species, J. Mol. Struct., 2017, 1141, 634-642.

8 A. Ioannou and C. Varotsis, Modifications of hemoglobin and myoglobin by Maillard reaction products (MRPs), PLoS One, 2017, 12, e0188095.

9 R. E. Hirsch and R. Nagel, Conformational studies of Hemoglobin using intrinsic Fluorescence measurements, $J$. Biol. Chem., 1981, 256, 1080-1083.

10 L. Sokolov and I. Mukerji, Conformational Changes in FmetHbS propped with UV Resonance Raman and Fluorescence spectroscopic methods, J. Phys. Chem. B, 1998, 102, 8314-8319.

11 B. Sengupta, S. Chakraborty, M. Crawford, J. M. Taylor, L. E. Blackmon, P. K. Biswas and W. H. Kramer, Characterization of diadzein-hemoglobin binding using optical spectroscopy and molecular dynamics simulations, Int. J. Biol. Macromol., 2012, 51, 250-258.

$12 \mathrm{~J}$. Leclère and I. Birlouez-Aragon, The fluorescence of advanced Maillard products is a good indicator of lysine damage during the Maillard reaction, J. Agric. Food Chem., 2001, 49, 4682-4687.

13 S. B. Matiacevich and M. P. Buera, A critical evaluation of fluorescence as a potential marker for the Maillard reaction, Food Chem., 2006, 95, 423-430.

14 I. N. Shipanova, M. A. Glomb and R. H. Nagaraj, Protein modification by methylglyoxal: chemical nature and synthetic mechanism of a major fluorescent adduct, Arch. Biochem. Biophys, 1997, 344, 29-36.

15 S. B. Matiacevich, P. R. Santagapita and M. P. Buera, Fluorescence from the Maillard reaction and its potential applications in food science, Crit. Rev. Food Sci. Nutr., 2005, 45, 483-495.

16 J. R. Lakowicz, Protein fluorescence, in Principles of fluorescence spectroscopy, Springer, Boston, MA, 1983, pp. 341-381.

17 J. P. Padilla-Martinez, W. Lewis, A. Ortega-Martinez and W. Franco, Intrinsic fluorescence and mechanical testing of articular cartilage in human patients with osteoarthritis, J. Biophotonics, 2018, 11(1), e201600269.

18 S. Chakraborty, S. Chaudhuri, B. Pahari, J. Taylor, P. K. Sengupta and B. Sengupta, A critical study on the interactions of hesperitin with human hemoglobin: Fluorescence spectroscopic and molecular modeling approach, J. Lumin., 2012, 132, 1522-1528.

19 M. R. Mihailescu, C. Fronticelli and I. M. Russu, Allosteric free energy changes at the $\alpha 1 \beta 2$ interface of human hemoglobin probed by proton exchange of $\operatorname{Trp} \beta 37$, Proteins: Struct., Funct., Bioinf., 2001, 44, 73-78.

20 V. Sugawara, E. Kadono, A. Suzuki, Y. Yukuta, Y. Shibasaki, N. Nishimura, Y. Kameyama, M. Hirota, C. Ishida, N. Higuchi and K. Haramoto, Hemichrome formation observed in human haemoglobin A under various buffer conditions, Acta Physiol. Scand., 2003, 179, 49-59.

21 A. Vergara, L. Vitagliano, G. Di Prisco, C. Verde and L. Mazzarella, Spectroscopic and crystallographic characterization of hemichromes in tetrameric hemoglobins, Methods Enzymol., 2008, 436A, 421-440.

22 V. L. Robinson, B. B. Smith and A. Arnone, A pH-Dependent Aquomet-to-Hemichrome Transition in Crystalline Horse Methemoglobin, Biochemistry, 2003, 42, 10113-10125.

23 M. Nagai, S. Kaminaka, Y. Ohba, Y. Nagai, Y. Mizutani and T. Kitagawa, Ultraviolet Resonance Raman Studies of Quaternary Structure of Hemoglobin Using a Tryptophan 37 Mutant, J. Biol. Chem., 1995, 270, 1636-1642.

24 Y. Dou, S. J. Admiraal, M. Ikeda-Saito, S. Krzywda, A. J. Wilkinson, T. Li, J. S. Olson, R. C. Prince, I. J. Pickering and G. N. George, Alteration of Axial Coordination by Protein Engineering in Myoglobin Bisimidazole Ligation in the $\mathrm{His}^{64} \rightarrow \mathrm{Val}^{6} \mathrm{Val}^{68} \rightarrow \mathrm{His}$ double mutant, J. Biol. Chem., 1995, 270, 15993-16001.

25 A. A. Booth, R. G. Khalifah, P. Todd and B. G. Hudson, In vitro kinetic studies of formation of antigenic advanced glycation end products (AGEs) novel inhibition of postAmadori glycation pathways, J. Biol. Chem., 1997, 272, 5430-5437.

26 C. Koutsoupakis, T. Soulimane and C. Varotsis, Docking site dynamics of $b a_{3}$-cytochrome $\mathrm{c}$ oxidase from Thermus thermophilus, J. Biol. Chem., 2003, 278, 36806-36809.

27 S. Stavrakis, E. Pinakoulaki, A. Urbani and C. Varotsis, Fourier transform infrared evidence for a ferric sixcoordinate nitrosylheme $b_{3}$ complex of cytochrome $c b b_{3}$ oxidase from Pseudomonas stutzeri at ambient temperature, J. Phys. Chem. B, 2002, 106, 12860-12862.

28 C. Varotsis and M. Vamvouka, Resonance Raman and Fourier Transform Infrared Detection of Azide Binding to the Binuclear Center of Cytochrome $b o_{3}$ Oxidase from Escherichia coli, J. Phys. Chem. B, 1999, 103, 3942-3946.

29 E. Pinakoulaki, H. Yoshimura, S. Yoshioka, S. Aono and C. Varotsis, Recognition and discrimination of gases by the oxygen-sensing signal transducer protein HemAT as revealed by FTIR spectroscopy, Biochemistry, 2006, 45, 7763-7766.

30 T. Ohta, E. Pinakoulaki, T. Soulimane, T. Kitagawa and C. Varotsis, Detection of a Photostable Five-Coordinate Heme $a_{3}-\mathrm{Fe}-\mathrm{CO}$ Species and Functional Implications of His384/ $\alpha 10$ in CO-Bound $b a_{3}$-Cytochrome $c$ Oxidase from Thermus thermophilus, J. Phys. Chem. B, 2004, 108, 54895491. 